\title{
Cellular Physiology

\section{The Electrophysiological Effects of Cardiac Glycosides in Human iPSC-derived Cardiomyocytes and in Guinea Pig Isolated Hearts}

\author{
Liang Guo ${ }^{1}$, Jian-Yong Qian"1, Rory Abrams ${ }^{1}$, Hai-Ming Tang ${ }^{2}$, \\ Thomas Weiser ${ }^{1}$, Martin J. Sanders ${ }^{2}$ and Kyle L. Kolaja ${ }^{1}$
}

${ }^{1}$ Global Early \& Investigative Safety, ${ }^{2}$ Safety Pharmacology, Non-Clinical Safety, Hoffmann-La Roche Inc., Nutley NJ

\section{Key Words}

Induced pluripotent stem cell (iPSC) • Cardiomyocyte - Langendorff isolated heart $\cdot$ Cardiac glycoside $\cdot \mathrm{Ca}^{2+}$ cycling • Contractility • Arrhythmia - Cardiotoxicity • Microelectrode array (MEA)

\begin{abstract}
Background/aims: Monitoring changes in the field potential (FP) of human induced pluripotent stem cellderived cardiomyocytes (hiPSC-CMs) following compound administration has been proposed as a novel screening tool to evaluate cardiac ion channel interactions and QT liability. Here we extended the use of FP to evaluate the pharmacological and toxicological properties of cardiac glycosides. Methods: FPs were recorded using microelectrode arrays (MEAs) in spontaneously beating hiPSC-CMs. The in vitro effects of ouabain and digoxin on FPs were compared with data generated on hemodynamic and ECG parameters in guinea pig Langendorff hearts. Results: In hiPSC-CMs, ouabain and digoxin reduced $\mathrm{Na}^{+}$-spike amplitude, shortened FP duration (FPD), increased $\mathrm{Ca}^{2+}$-wave amplitude, and dosedependently induced arrhythmic beats. The ouabaininduced changes observed in hiPSC-CMs correlated
\end{abstract}

\section{KARGER}

Fax +4161306 1234

E-Mail karger@karger.ch

www.karger.com
(C) 2011 S. Karger AG, Basel

1015-8987/11/0275-0453\$38.00/0

Accessible online at:

www.karger.com/cpb well with the effects seen in isolated hearts which revealed QT shortening, enhancement of contractility, and arrhythmogenesis. Nifedipine, an L-type $\mathrm{Ca}^{2+}$ channel blocker, reduced $\mathrm{Ca}^{2+}$-wave amplitude and FPD in hiPSC-CMs, and led to parallel effects of decreased ventricular contractility and shortened QT interval in isolated hearts. Further, nifedipine attenuated the $\mathrm{Ca}^{2+}$-peak amplitude and proarrhythmic effect of both glycosides. These results suggested that FPD and $\mathrm{Ca}^{2+}$-wave amplitude are comparable surrogates of QT interval and contractility of intact hearts, respectively. Conclusion: hiPSC-CMs reflect similar cardiac pharmacology as seen in isolated cardiac preparations and thus are a suitable model in study of the pharmacology and toxicology of cardioactive ion channel and transporter modulators.

Copyright $\odot 2011$ S. Karger AG, Basel

\section{Introduction}

Unanticipated safety findings in drug development are the main reason for late-stage attrition [1]. The paucity of a predictive in vitro model reflective of cardiac biology in the current toolkit in drug safety evaluation highlights

Kyle L. Kolaja, Ph.D., D.A.B.T., Fellow, A.T.S.

Hoffmann-La Roche, Inc. Bldg. 100/325

340 Kingsland Street, Nutley NJ 07110 (USA)

Tel. +1-973-235-4077, Fax +1-973-235-4710

E-Mailkyle.kolaja@roche.com 
the need to identify a new approach to screen drug candidates. A sound drug safety profiling strategy requires implementation of in vitro models that predict accurately the outcomes seen in traditional safety pharmacology models (ICH S7A and S7B) [2]. Hence, human in vitro models must be validated against well-defined in vivo end-points if these assays are expected to be a part of the future drug safety testing paradigm [3].

Cardiovascular toxicity has been a leading cause of marketed drug withdrawal, restriction of clinical use, and delay or denial of regulatory approval over the last decade. In a recent survey, 17 out of 51 (33\%) safety-based drug withdrawals between 1998-2008 in North American, European and Asian markets were attributed to cardiovascular adverse effects [4]. This emphasizes the need to implement novel in vitro assays that are predictive of cardiovascular side effects.

Recent progress in stem cell technology has improved the ability to produce functional human cardiomyocytes in a large-scale and of high quality, making it feasible for routine screening of cardiac drug safety directly in human cardiomyocytes [5-7]. One technology capable of detecting the rhythmic change of electric potentials generated by beating hiPSC-CMs is the microelectrode array (MEA) system. This system in combination with hiPSC-CMs accurately characterized the effects of several known inhibitors of the major cardiac ion channels (e.g. $\mathrm{Na}^{+}$, L-type $\mathrm{Ca}^{2+}$, hERG and $\mathrm{I}_{\mathrm{Ks}}$ ) as well as the action of modulators of $\alpha, \beta$-adrenergic and muscarinic receptors [8-10]. These results demonstrate the value of MEA as a novel cellular electrophysiology platform suitable to screen for deleterious cardioactive properties of drugs in discovery [11-13].

The cardiac glycosides including ouabain and digitalis are a class of compounds that selectively inhibit $\mathrm{Na}^{+} / \mathrm{K}^{+}-$ ATPase, and lead to an increase in cardiac contractility. This pharmacological property has been shown to be beneficial in the treatment of heart failure. However, one clinical side effect of cardiac glycoside treatment is the provocation of severe ventricular arrhythmia via dysregulation of transmembrane $\mathrm{Na}^{+}$and $\mathrm{Ca}^{2+}$ cycling [14] This type of arrhythmia is distinguished from those associated with hERG channel inhibition and QT prolongation [15]. To investigate whether both pharmacological and toxicological effects of cardiac glycosides can be identified in hiPSC-CMs by MEA, two cardiac glycosides, ouabain and digoxin, were tested. In addition, the antagonizing action of nifedipine, a potent $\mathrm{L}$ type $\mathrm{Ca}^{2+}$ channel antagonist, on both cardiac glycosides was examined in hiPSC-CMs. To confirm and assess the predictivity of the in vitro model, the functional translation of FP parameters, ouabain and nifedipine were further tested in the Langendorff isolated hearts.

\section{Materials and Methods}

\section{Cell culture}

Cryopreserved human iPSC-derived cardiomyocytes (iCells) from Cellular Dynamics International (CDI, Madison, WI) were thawed in Plating Media (CDI) and plated onto $0.1 \%$ Gelatin (Sigma, St. Louis, MO)-coated, 6-well tissue-culture plates (Corning, Edison, NJ) at a density of 1.5-2.5 x 10 cells per well. Cells were cultured for $6-10$ days at $37{ }^{\circ} \mathrm{C}, 7 \% \mathrm{CO}_{2}$ prior to re-plating onto the microelectrode arrays (MEAs, Multichannel Systems, Reutlingen, Germany). The media was changed every 2 days after plating using Maintenance Media (CDI).

MEAs were prepared according to manufacturer guidelines. Briefly, the recording area of 6-well MEA dishes (6wellMEA200/30iR-Ti-mr, Multichannel Systems) was coated with $2 \mu \mathrm{L}$ Fibronectin (Sigma) diluted 1:20 and incubated at 37 ${ }^{\circ} \mathrm{C}$ for 3 hours. Cells were dissociated from 6 -well plates using $0.5 \%$ trypsin-EDTA (Invitrogen, Carlsbad, CA) and reseeded at the target density of $3 \times 10^{4}$ cells in a $2 \mu \mathrm{L}$ delivery to each recording well, and incubated at $37{ }^{\circ} \mathrm{C}, 7 \% \mathrm{CO}_{2}$ for 3 hours prior to filling each well with Maintenance Media. The media of the wells in the MEAs were changed every 2 days thereafter with Maintenance Media pre-warmed to $37^{\circ} \mathrm{C}$. Cells were cultured for 3 to 6 days until a spontaneously beating syncytial monolayer was formed prior to the conduction of experiments.

\section{MEA assay}

Experiments were conducted in a customized, environmentally controlled chamber perfused with a $95 \% \mathrm{O}_{2}$ and $5 \%$ $\mathrm{CO}_{2}$ at $37 \pm 0.1{ }^{\circ} \mathrm{C}$. FPs from spontaneously beating cardiomyocytes were recorded and digitized at $10 \mathrm{KHz}$ using MEA60 data acquisition system (Multichannel Systems). The drug stocks were made in DMSO at 1000-fold of highest testing concentration which was then serially diluted in Maintenance Media to 20x the target concentrations. Following an equilibration period of 15 minutes, the drug was added directly to the bath in a cumulative manner. Up to 6 concentrations were tested in each MEA recording dish with $\sim 10$ minute drug exposure for each concentration. All drugs were tested as $n \geq 4$ wells. Each 6-well MEA possessed at least one well as the timematched vehicle (DMSO) control.

\section{Langendorff isolated heart assay}

The procedure was similar to that described previously [16]. Briefly, the heart of adult guinea pigs weighing 450-550g was isolated and retrogradely perfused with Krebs-Henseleit (KH) buffer at the constant pressure of $85 \mathrm{mmHg}$ on the Langendorff apparatus. The coronary perfusion rate (CPR), left ventricular developed pressure (LVDP) and electrocardiogram (ECG) were monitored and recorded continuously by Ponemah acquisition system (Version 4.9, DSI,

Guo/Qian/Abrams/Tang/Weiser/Sanders/Kolaja 


\begin{tabular}{lcccccc}
\hline Parameters & $\begin{array}{c}\text { Beat rate } \\
(\text { beat/min })\end{array}$ & $\begin{array}{c}\mathrm{Na}^{+} \text {-spike } \\
(\mu \mathrm{V})\end{array}$ & $\begin{array}{c}\mathrm{Ca}^{2+} \text {-wave } \\
(\mu \mathrm{V})\end{array}$ & $\begin{array}{c}\text { RR interval } \\
(\mathrm{ms})\end{array}$ & $\begin{array}{c}\mathrm{FPD} \\
(\mathrm{ms})\end{array}$ & $\begin{array}{c}\mathrm{FPD}_{\text {cf }} \\
(\mathrm{ms})\end{array}$ \\
\hline Baseline & $45 \pm 2$ & $743 \pm 183$ & $46 \pm 7$ & $1355 \pm 77$ & $565 \pm 50$ & $511 \pm 43$ \\
Vehicle \#1 & $46 \pm 2$ & $755 \pm 211$ & $45 \pm 6$ & $1330 \pm 61$ & $570 \pm 52$ & $512 \pm 44$ \\
Vehicle \#2 & $46 \pm 2$ & $773 \pm 213$ & $45 \pm 6$ & $1326 \pm 49$ & $573 \pm 49$ & $521 \pm 42$ \\
Vehicle \#3 & $46 \pm 2$ & $751 \pm 203$ & $45 \pm 7$ & $1325 \pm 50$ & $574 \pm 51$ & $522 \pm 43$ \\
Vehicle \#4 & $44 \pm 2$ & $763 \pm 214$ & $45 \pm 6$ & $1362 \pm 47$ & $584 \pm 46$ & $527 \pm 40$ \\
Vehicle \#5 & $46 \pm 2$ & $763 \pm 200$ & $44 \pm 7$ & $1320 \pm 46$ & $576 \pm 51$ & $524 \pm 43$ \\
Vehicle \#6 & $45 \pm 2$ & $767 \pm 192$ & $46 \pm 7$ & $1330 \pm 46$ & $573 \pm 53$ & $521 \pm 56$ \\
\hline
\end{tabular}

Table 1. Stability of measured FP parameters during exposure to the vehicle $(0.1 \%$ DMSO) throughout the experiment. Vehicle \#1-\#6 indicates the sequence of recording time sections corresponding to those for drug testing. $n=6$ wells.

St. Paul, MN). Hearts beat spontaneously under the sinus rhythm except at the end of each treatment when a brief pacing at $230 \mathrm{beat} / \mathrm{min}$ was applied. The $\mathrm{KH}$ buffer contained (in $\mathrm{mM}$ ) $\mathrm{NaCl} 120, \mathrm{KCl} 4.7, \mathrm{MgSO}_{4} 1.2, \mathrm{NaHCO}_{3} 25.0, \mathrm{KH}_{2} \mathrm{PO}_{4} 1.2$, glucose 11.1, Na-pyruvate 2.0 and $\mathrm{CaCl}_{2} 1.25$, was warmed to $(36.5 \pm 1$ ${ }^{\circ} \mathrm{C}$ ) and equilibrated with a mixture of $95 \% \mathrm{O}_{2}$ and $5 \% \mathrm{CO}_{2}$ to maintain the $\mathrm{pH}$ at $7.35-7.40$. The experiment started after a period of $\geq 25$ minute stabilization. Each heart was first perfused with the vehicle ( $\leq 0.3 \%$ DMSO), then the drug with $3-4$ ascending concentrations each for 15 minutes. Each drug was tested in 4 hearts.

\section{Data analysis}

Data were first analyzed using the built-in analysis modules of Ponemah (DSI) and MEA systems (Multichannel Systems). Initial results were then exported into in-house developed Microsoft Excel templates for further semiautomated analysis. For MEA, at least 20 beats towards the end of drug exposure at each concentration or prior to the occurrence of asynchronous contractions were analyzed or averaged. The baseline measurement was taken from the last minute of equilibration period. The " $\mathrm{Na}^{+}$"-spike and " $\mathrm{Ca}^{2+}$ "waves were represented in the field potential complex as defined by the initial rapid spike and the subsequent slow return-tothe baseline amplitude, respectively. The rate of synchronous beats was calculated by the number of discrete " $\mathrm{Na}^{+}$"-spikes detected during one minute. The " $\mathrm{Na}^{+"}$ "-spike amplitude was measured automatically with a computerized algorithm that identified both the positive and negative peak of a spike. The point upon which the slow " $\mathrm{Ca}^{2+}$ "-wave returned to baseline or reached its peak in case of the formation of a biphasic waveform was identified manually. The difference in potential between the positive peak and the negative peak of the " $\mathrm{Ca}^{2+}$ "-wave was defined as the " $\mathrm{Ca}^{2+"}$-wave amplitude. The length of time difference between " $\mathrm{Na}^{+}$"-spike and the end of " $\mathrm{Ca}^{2+}$ "-amplitude was measured as the FPD. The averaged waveforms at each concentration were overlapped (see Figs. 1, 4 and 6 ) and the initial measurement was verified manually by a second, independent investigator. For the isolated heart experiments, four consecutive beats prior to the occurrence of

The Electrophysiological Effect of Cardiac Glycosides severe arrhythmia or during the final minute of drug exposure at each concentration were measured. The drug responses were similar in sinus rhythm or under pacing in the isolated hearts preparations, thus only the data obtained from the sinus rate are presented.

To avoid the influence of beat rate on FPD and QT interval, the raw measurement of both FPD and QT interval was corrected by the spontaneous beating rate using Fridericia's formula: FPDcf or QTcf = FPD or QT/RR ${ }^{1 / 3}$, whereas RR represents the beat-to-beat interval, FPDcf and QTcf are FPD and QT corrected by Fridericia's formula.

\section{Statistics}

To assess the treatment-related changes for each parameter, the measurement after treatment was calculated as $\%$ change from the baseline (pre-drug) level, and compared to that obtained from the time-matched vehicle control group. Data were expressed as the mean \pm standard error, and the statistical significance of the differences was analyzed with a one-way ANOVA followed by Dunnett's multiple comparison test using JMP (Version 8.0.1., SAS Institute, Cary, NC). A pvalue $<0.05$ was considered statistically significant.

\section{Results}

\section{Parameters and stability of $F P$ recordings in human iPSC-CMs}

A typical FP from MEA consists of two components: a rapid, bi-directional spike followed by a slow, negativelyrunning wave which returns gradually towards the baseline level (Fig. 1A and 1B, left). These two waveform features are termed the " $\mathrm{Na}^{+}$-spike" and " $\mathrm{Ca}^{2+}$-wave" since they are mainly mediated by the transmembrane $\mathrm{Na}^{+}$and $\mathrm{Ca}^{2+}$ influx [17], respectively. As illustrated in Fig. 1B, when hiPSC-CMs were exposed to the vehicle $(0.1 \%$ DMSO), FPs remained stable throughout the $\sim 120$ minute experiment period. The stability of the system was 
Fig. 1. Effects of ouabain and digoxin on FPs in hiPSC-CMs. (A) Screenshots of representative raw tracings to illustrate the dose-dependent morphological change of FPs caused by ouabain, and the development of ventricular tachycardia (VT)-like and ventricular fibrillation (VF)-like arrhythmic beats. (B) Overlaps of averaged traces (from $\geq 20$ traces at each concentration) to demonstrate the stability of FPs throughout the entire experiment period when exposed to the vehicle, and the dose-dependent change of FPs when the cells were exposed to ouabain and digoxin at the escalated concentrations. \#1-\#6 represented the averaged traces of vehicle treatment in the time span matching that of drug exposure at each ascending doses. Note, averaged traces are not available at $10 \mu \mathrm{M}$ ouabain due to severe VFs developed in all wells at end of exposure to $3 \mu \mathrm{M}$.

Fig. 2. Dose-response plots to show the effects of the vehicle ( $0.1 \%$ DMSO), ouabain and digoxin on the spontaneous beat rate, $\mathrm{Na}^{+}$-spike amplitude, FP duration corrected by the beat rate with Fridericia's formula (FPDcf) and $\mathrm{Ca}^{2+}$-wave amplitude. All data were normalized to represent the percentage $(\%)$ change from the baseline control value and $\mathrm{n}=6$ wells for all three groups. * denoted $P<0.05$, by compared with the timematched vehicle control group. Note, data at 10 $\mu \mathrm{M}$ ouabain were not available due to occurrence of VF in all wells at end of exposure to $3 \mu \mathrm{M}$.
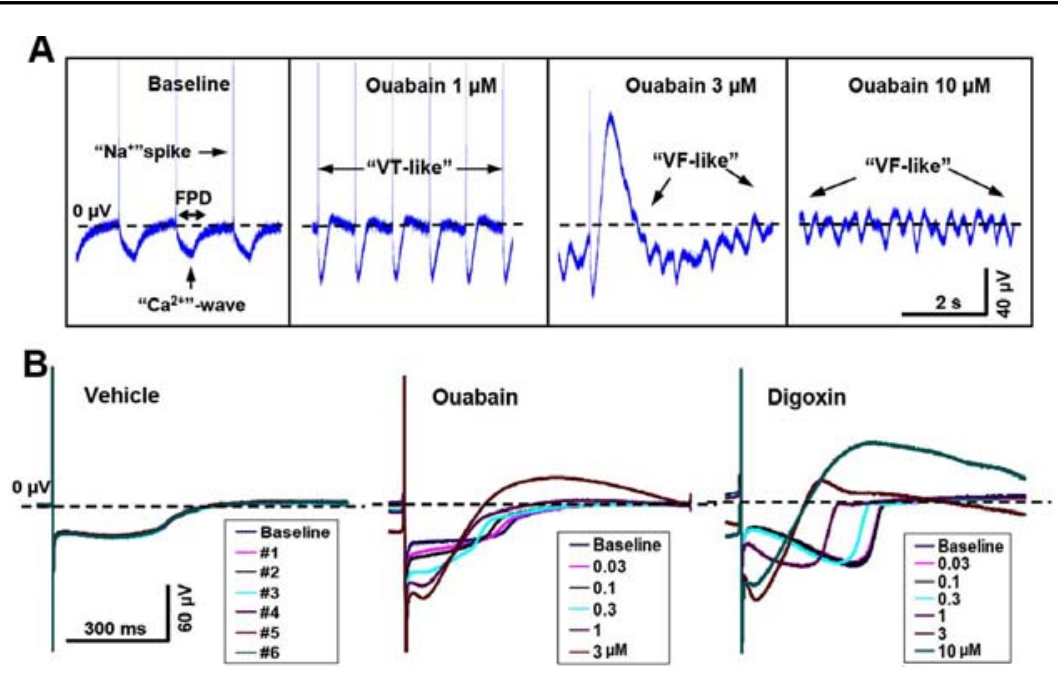

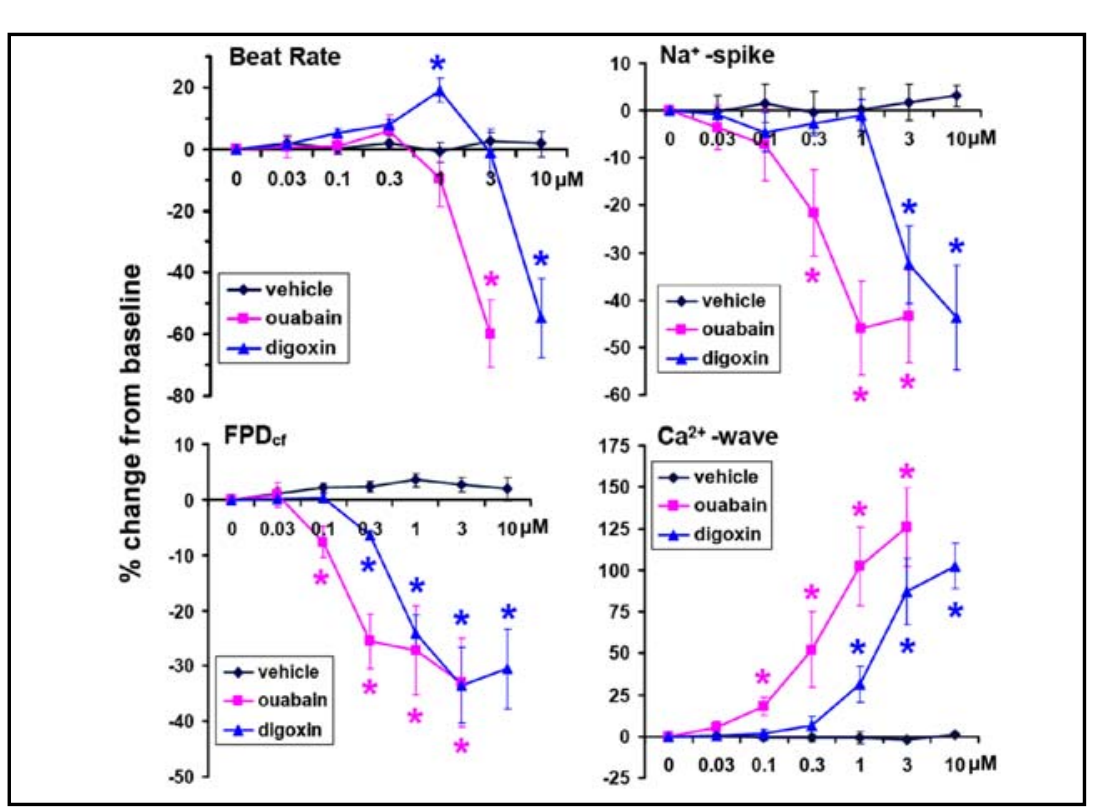

demonstrated through measurements taken from 6 wells across 4 different MEA recording dishes which did not exhibit any significant changes on all 6 parameters assessed (Table 1).

\section{Effects of cardiac glycosides on human iPSC- CMs}

Application of either ouabain or digoxin to hiPSC$\mathrm{CMs}$ reduced the $\mathrm{Na}^{+}$-spike amplitude and shortened the FPDcf, but increased the $\mathrm{Ca}^{2+}$-wave amplitude in a timeand dose-dependent manner (Fig. 1 and 2). Initial increases in $\mathrm{Ca}^{2+}$-wave amplitude were often accompanied by a transient acceleration of spontaneous beats, called "ventricular tachycardia (VT)-like beats" as they were reminiscent of the sustained VT in a heart. With time, the rate of synchronous beats declined rapidly and were

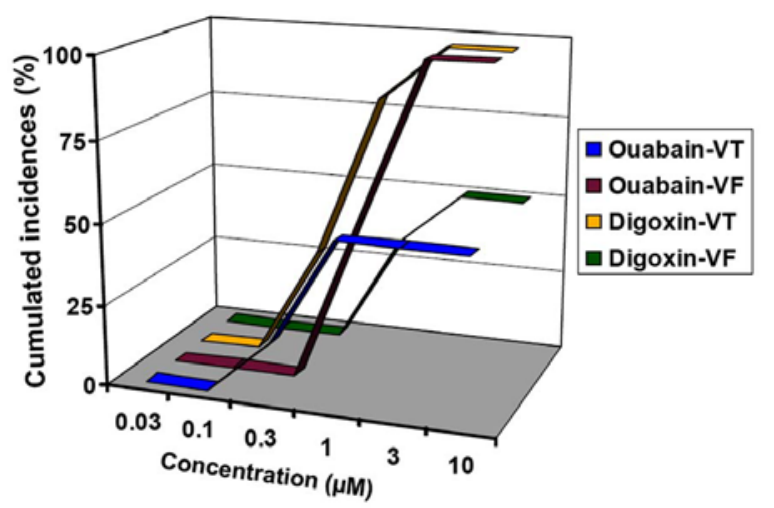

Fig. 3. Cumulative plot to illustrate the dose-dependent increase in the incidences of ouabain and digoxin-induced ventricular tachycardia (VT)- and fibrillation (VF)-like arrhythmic events. Note, such arrhythmic events were never observed in cardiomyocytes exposed to the vehicle.

Guo/Qian/Abrams/Tang/Weiser/Sanders/Kolaja 
Fig. 4. Effects of ouabain on isolated guinea pig Langendorff hearts. (A) Screenshots of representative raw tracings to illustrate the change of left-ventricle developed pressure (LVDP), $\pm \mathrm{dP} /$ $\mathrm{dt}$ and ECG caused by ouabain. (B) Dose-response plots to show the effects of the vehicle $(0.1 \%$ DMSO) and ouabain on QT interval, corrected by the heart rate with Fridericia's formula (QTcf), LVDP and $+\mathrm{dP} / \mathrm{dt}$. All data were normalized to the baseline value and $\mathrm{n}=4$ hearts for both groups. * denoted $P<0.05$, by compared with the time-matched vehicle control group. (C) Screenshots of representative raw tracings to illustrate the development of ventricular ectopic beats, tachycardia and fibrillation when the heart was exposure to $1 \mu \mathrm{M}$ ouabain. Arrow-heads denote the single or short-run ventricular ectopic beats; Note, the rapid transition of sustained VTs to VFs.

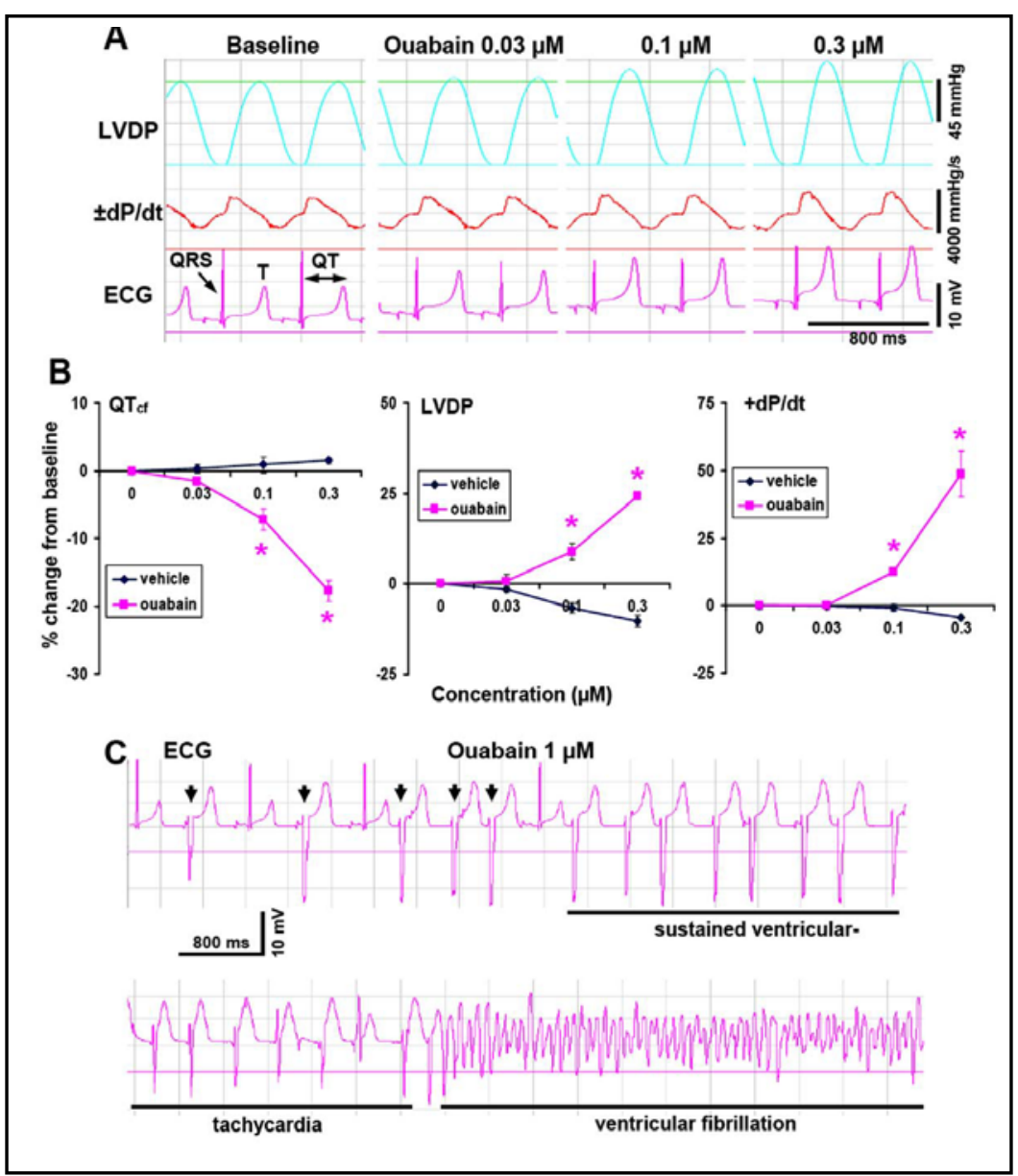

\begin{tabular}{lrccccc}
\hline Concentration $(\mu \mathrm{M})$ & & 0.03 & 0.1 & 0.3 & 1 & 3 \\
\hline MEA & $\mathrm{FPD}_{\mathrm{cf}}$ & 1 & -8 & -26 & -27 & -33 \\
Isolated-heart & $\mathrm{QT}_{\mathrm{cf}}$ & -2 & -7 & -18 & $\mathrm{NA}$ & $\mathrm{NT}$ \\
MEA & & & & & & \\
Isolated-heart & $\mathrm{Ca}^{2+}-\mathrm{wave}$ & 5 & 18 & 52 & 102 & 126 \\
MEA & $+\mathrm{dP} / \mathrm{dt}$ & 0 & 13 & 49 & $\mathrm{NA}$ & $\mathrm{NT}$ \\
Isolated-heart & arrhytmia & $\mathrm{NO}$ & $\mathrm{NO}$ & $\mathrm{VT}(1 / 6)$ & $\mathrm{VT}(2 / 6)$ & $\mathrm{VF}(6 / 6)$ \\
& arrhytmia & $\mathrm{NO}$ & $\mathrm{NO}$ & $\mathrm{VT}(2 / 4)$ & $\mathrm{VT}(2 / 6)$ & $\mathrm{NT}$ \\
\hline
\end{tabular}

Table 2. Comparison of ouabain-induced changes in hiPSC-CMs with those in the Langendorff isolated hearts. Values in the table represent the mean of \% changes caused by ouabain from the baseline value in 6 MEA wells or 4 isolated hearts, and the number of wells or hearts that exhibited VT- and/or VF-like arrhythmia. NA, not available; NT, not tested; VT, ventricular tachycardia; VF, ventricular fibrillation. entirely absent with full development of asynchronous, small-amplitude, high-frequency waves, termed "ventricular fibrillation (VF)-like beats" due to their resemblance to the VFs seen in the isolated heart preparations. The dose-dependent effects of ouabain and digoxin on the field potential waveform are illustrated by overlapping the averaged MEA traces from each concentration tested (Fig 1B). Since VF-like beats developed in all wells at end of exposure to $3 \mu \mathrm{M}$ ouabain, analysis at $10 \mu \mathrm{M}$ ouabain was not possible. Due to the occurrence of VT-like beats at $0.3 \mu \mathrm{M}$ ouabain and $1 \mu \mathrm{M}$ digoxin, an increase in the beat rate was observed at these respective concentrations prior to a quick decline in the synchronous beats at higher concentrations (Fig 2). $3 \mu \mathrm{M}$ ouabain reduced $\mathrm{Na}^{+}$-spike amplitude by $\sim 46 \%$ from the baseline value, FPDcf by $\sim 33 \%$ and increased $\mathrm{Ca}^{2+}$-wave 
Fig. 5. Effects of nifedipine on FPs in hiPSCCMs. (A) Screenshots of representative raw tracings to illustrate the morphological and beat rate change of FPs in the presence of nifedipine. (B) Overlaps of averaged traces (from $\geq 20$ traces at each concentration) to demonstrate the dose-dependent reduction of $\mathrm{Ca}^{2+}$-wave amplitude and FP duration. (C) Dose-response plots to show the change of beat rate, FPDcf and $\mathrm{Ca}^{2+}$-wave amplitude. $\mathrm{n}$ $=4$ wells. ${ }^{*}$ denoted $P<0.05$, by compared with the time-matched vehicle control group. guinea pig Langendorff hearts. (A) Screenshots of representative raw tracings to illustrate the change of coronary flow rate (CFR), left-ventricle developed pressure (LVDP), $\pm \mathrm{dP} / \mathrm{dt}$ and ECG in the presence of nifedipine. (B) Dose-response plots to show the effects of nifedipine on CFR, QTcf, LVDP and $+\mathrm{dP} / \mathrm{dt}$. All data were normalized to the baseline value and $\mathrm{n}=4$ hearts. ${ }^{*}$ denoted $P<0.05$, by compared with the time-matched vehicle control group.
Fig. 6. Effects of nifedipine on isolated
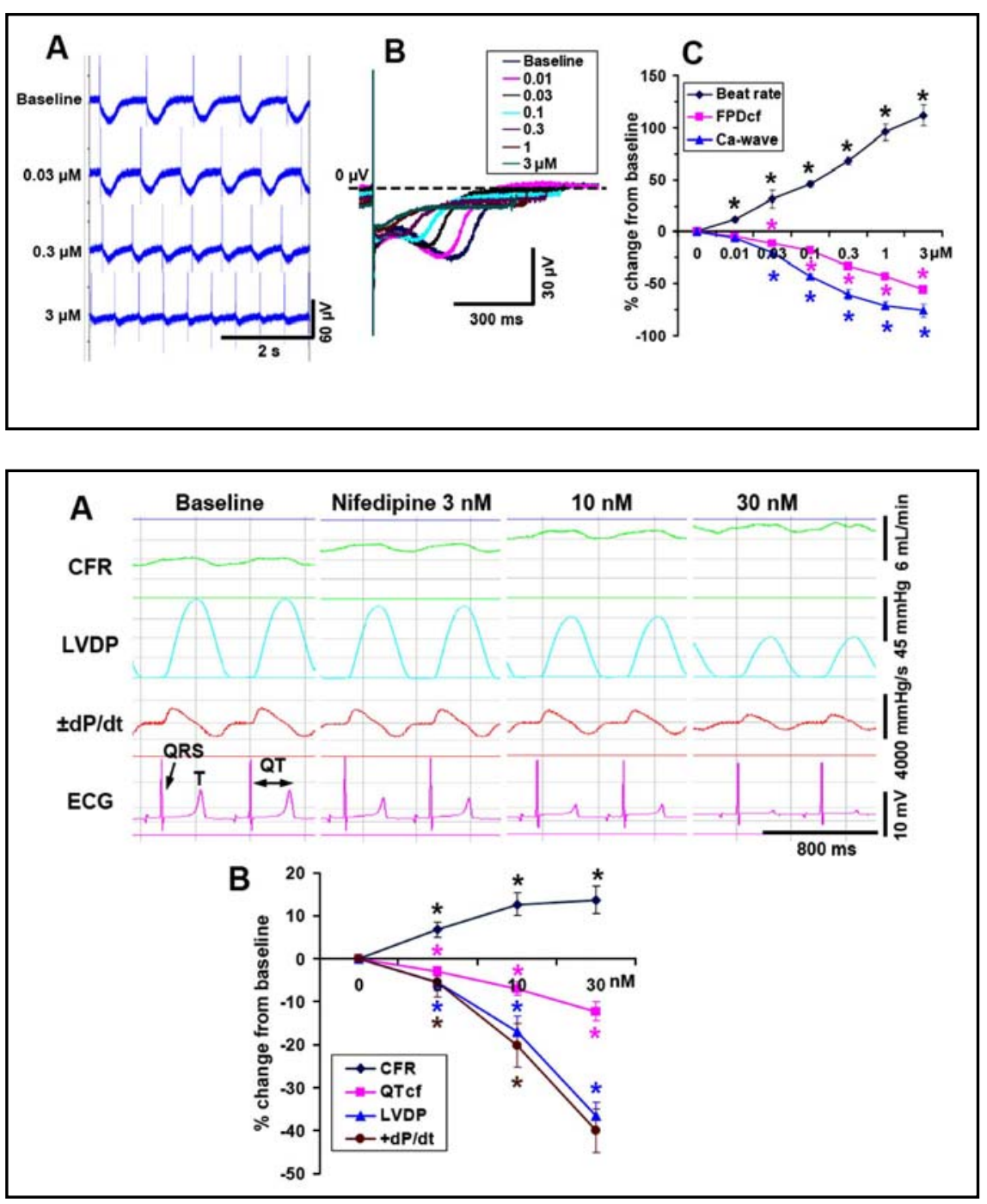

Fig. 7. Blockade of cardiac glycosides' effects on $\mathrm{Ca}^{2+}$-wave amplitude by nifedipine. Overlaps of averaged traces to illustrate the change of $\mathrm{Ca}^{2+}$-wave amplitude caused by 1 $\mu \mathrm{M}$ ouabain alone (A), concurrent application of $30 \mathrm{nM}$ nifedipine with $1 \mu \mathrm{M}$ ouabain (B) and pre-treatment of cells with $30 \mathrm{nM}$ nifedipine for 5 minutes $(\mathrm{C})$ prior to addition of $1 \mu \mathrm{M}$ ouabain. Ouabain (D) and digoxin (E)-induced elevation in $\mathrm{Ca}^{2+}$-wave amplitude was mitigated by concurrent application (middle bar) or pre-treatment (right bar) with nifedipine. ${ }^{*}$ denoted $P<$ 0.05 , compared with that obtained by ouabain or digoxin alone. ctrl (vehicle control), oua (ouabain), nif (nifedipine).

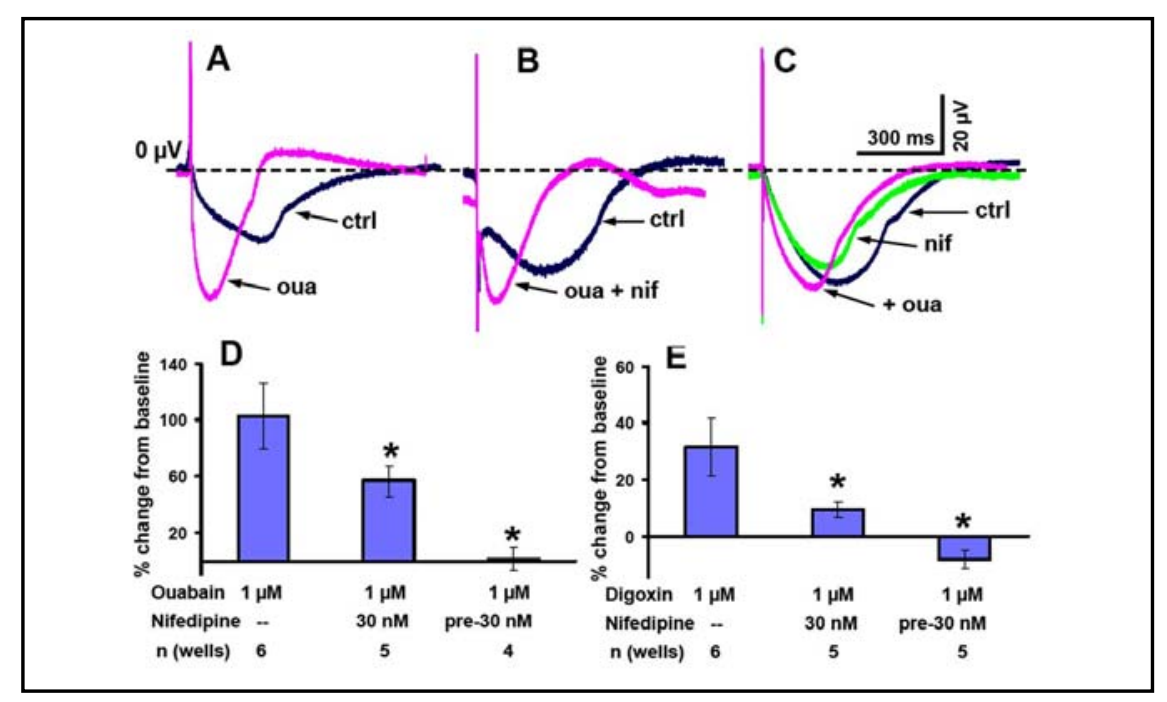

amplitude by $\sim 126 \%$. The corresponding dataset for digoxin with concentrations tested up to $10 \mu \mathrm{M}$ was $\sim 44 \%, \sim 34 \%$ and $\sim 103 \%$, respectively, suggesting that ouabain was $\sim 3$-fold more potent than digoxin. Arrhythmic beats occurred at concentrations $\geq 0.3 \mu \mathrm{M}$ for both

cardiac glycosides. Typically, arrhythmic episodes were first seen as a transient, VT-like beat pattern, which was followed by the rapid decline of the synchronized contraction and the development of rapid VF-style beats (Fig. 1A). VF-like beats developed eventually in all wells

Guo/Qian/Abrams/Tang/Weiser/Sanders/Kolaja 


\begin{tabular}{lrccccccc}
\hline Concentration $(\mu \mathrm{M})$ & & 0.003 & 0.01 & 0.03 & 0.1 & 0.3 & 1 & 3 \\
\hline MEA & $\mathrm{FPD}_{\mathrm{cf}}$ & $\mathrm{NT}$ & -8 & -19 & -28 & -44 & -54 & -65 \\
Isolated-heart & $\mathrm{QT}_{\mathrm{cf}}$ & -3 & -7 & -12 & $\mathrm{NT}$ & $\mathrm{NT}$ & $\mathrm{NT}$ & $\mathrm{NT}$ \\
MEA & $\mathrm{Ca}^{2+}-$ wave & $\mathrm{NT}$ & -6 & -20 & -43 & -61 & -71 & -76 \\
Isolated-heart & $+\mathrm{dP} / \mathrm{dt}$ & -6 & -20 & -40 & $\mathrm{NT}$ & $\mathrm{NT}$ & $\mathrm{NT}$ & $\mathrm{NT}$ \\
\hline
\end{tabular}

Table 3. Comparison of nifedipine-induced changes in hiPSC-CMs with those in the Langendorff isolated hearts. Values in the table represent the mean of \% changes caused by ouabain from the baseline value in 4 MEA wells or 4 isolated hearts. NT, not tested.

that were exposed to ouabain (Table 2) or digoxin at 10 $\mu \mathrm{M}$. The dose-dependent increase in the incidences of VT- and VF-like beats produced by ouabain and digoxin are shown in the cumulative plot (Fig. 3).

Effects of ouabain on isolated guinea pig hearts

As shown in the isolated guinea pig hearts (Fig. 4), ouabain dose-dependently shortened the QT interval (QTcf ) by up to $18 \%$ from the baseline and increased the left-ventricular developed pressure (LVDP, up to 24\%) and its maximal rate of rise $(+\mathrm{dP} / \mathrm{dt}$, up to $49 \%)$. Arrhythmia was induced at concentrations $\geq 0.3 \mu \mathrm{M}$, and typically, the onset of arrhythmia started with a gradual increase in incidence of sporadic ectopic ventricular contractions, followed by a short-train or sustained VT which occasionally would deteriorate into VF (Fig. 4C).

\section{Effects of nifedipine on human iPSC-CMs}

Nifedipine at concentrations between 0.01 and $3 \mu \mathrm{M}$ dose-dependently accelerated the spontaneous beat rate (by up to 112\% from the baseline), shortened FPDcf (up to $56 \%$ ) and reduced $\mathrm{Ca}^{2+}$-wave amplitude (up to $76 \%$ ) (Fig. 5). Nifedipine also caused a mild reduction $(\sim 20 \%)$ in $\mathrm{Na}^{+}$-spike amplitude at $3 \mu \mathrm{M}$ (data not shown).

\section{Effects of nifedipine on isolated guinea pig hearts}

In isolated Langendorff hearts (Fig. 6), at 3 to 30 $\mathrm{nM}$ nifedipine shortened QTcf (by up to $12 \%$ from the baseline), reduced LVDP (up to $37 \%$ ) and $+\mathrm{dP} / \mathrm{dt}$ (up to $40 \%$ ) in a dose-dependent manner. Nifedipine also increased the coronary flow rate by up to $14 \%$.

\section{Alleviation of cardiac glycoside effects by nifedipine}

To examine the anti-arrhythmic effect of nifedipine on cardiac glycosides, $1 \mu \mathrm{M}$ ouabain or digoxin was applied to cardiomyocytes either concurrently with $30 \mathrm{nM}$ nifedipine, or after the cells were pre-treated with $30 \mathrm{nM}$

The Electrophysiological Effect of Cardiac Glycosides nifedipine for 5 minutes. As shown in Fig. 7, $1 \mu \mathrm{M}$ ouabain alone increased $\mathrm{Ca}^{2+}$-wave amplitude by $\sim 102 \%$ from the baseline. When administrated concomitantly with 30 $\mathrm{nM}$ nifedipine, $1 \mu \mathrm{M}$ ouabain increased heart rate by $\sim 57 \%$ from the baseline. The effect of $1 \mu \mathrm{M}$ ouabain on $\mathrm{Ca}^{2+}$-wave amplitude was nearly abolished (as demonstrated by a $\sim 1 \%$ change from the baseline) when the cells were pre-treated with $30 \mathrm{nM}$ nifedipine for 5 minutes prior to ouabain addition. Similarly, $1 \mu \mathrm{M}$ digoxin increased $\mathrm{Ca}^{2+}$-wave amplitude by $\sim 31 \%$ and $9 \%$, respectively, when applied alone or together with $30 \mathrm{nM}$ nifedipine. Pre-treatment of human hiPSC-CMs with 30 $\mathrm{nM}$ of nifedipine for 5 minutes reduced $\mathrm{Ca}^{2+}$-wave amplitude by $\sim 20 \%$ from baseline, and this reduction was attenuated to a $\sim 8 \%$ decrease from baseline after subsequent addition of $1 \mu \mathrm{M}$ digoxin.

Nifedipine also prevented the induction of arrhythmic events by ouabain and digoxin. Application of $1 \mu \mathrm{M}$ ouabain alone induced VT-like beats in 2 wells and VFlike beats in 3 wells (out of 6 total wells). In contrast, in wells pre-treated with $30 \mathrm{nM}$ nifedipine, no VT- and VFlike beats were induced by $1 \mu \mathrm{M}$ ouabain, while simultaneous treatment of $1 \mu \mathrm{M}$ ouabain and $30 \mathrm{nM}$ nifedipine together led to only VT-like beats in one out of 5 wells exposed. Similarly, $1 \mu \mathrm{M}$ digoxin by itself caused VT-like beats in 5 wells and VF-like beats in 2 wells out of 6 wells tested. Pre-treatment with $30 \mathrm{nM}$ nifedipine prevented any instance of VT- and VF-like beats after subsequent administration of $1 \mu \mathrm{M}$ digoxin and simultaneous exposure to $1 \mu \mathrm{M}$ digoxin and $30 \mathrm{nM}$ nifedipine together led to one of 5 wells with VT-like beats.

\section{Discussion}

The high rate of drug failure due to safety liabilities during late stage development and post-marketing surveillance is a driving force for exploring, validating and implementing novel in vitro models $[1,4]$. High-throughput 
in vitro assays with robust predictive power towards validated endpoints will enable safety screening of virtually all discovery molecules, so that only molecules with optimal safety profiles can advance into development.

Access to functional human cardiomyocytes with an unlimited supply opens a new avenue to develop novel functional assays for early cardiac drug safety evaluation $[5,6]$. So far, several groups have employed MEA systems to characterize the electrophysiological, pharmacological and toxicological properties of hiPSC-CMs, with the proposal to develop a novel in vitro electrophysiological model for cardiac drug safety assessment, in particular, for the early screening of QT liability [7-11]. Using the MEA system with hiPSC-CMs, we have observed the expected electrophysiological response of hiPSC-CMs across a large panel of known cardiac ion channel and receptor blockers and modulators. Importantly, proarrhythmic drugs (e.g. E-4031, cisapride, and sotalol) that cause Torsade de pointes (TdP), a lethal polymorphous ventricular tachycardia, in the clinic reproducibly prolonged FPDcf and induced arrhythmic beating at concentrations relevant to the therapeutic exposure (unpublished data).

FPs recorded in cardiomyocytes by MEA reflect the highly-integrated activities of cardiac ion channels, exchangers and transporters. The initial rapid spike is produced mainly by the rapid $\mathrm{Na}^{+}$influx via $\mathrm{Na}^{+}$channels, whereas the $2^{\text {nd }}$ slow wave is mediated largely by the transmembrane cycling of $\mathrm{Ca}^{2+}$ [17] . Because drug-induced dysregulation of $\mathrm{Ca}^{2+}$ cycling can also lead to fatal ventricular arrhythmia in the clinic [18], we speculated whether the analysis of $\mathrm{Ca}^{2+}$-wave of FPs would be useful to detect this type of arrhythmia. Cardiac glycosides are well-known for exerting positive inotropy and inducing severe ventricular arrhythmia via altered transmembrane $\mathrm{Ca}^{2+}$ cycling [14]. In guinea pig ventricular cardiomyocytes, ouabain increased intracellular $\mathrm{Ca}^{2+}$ concentration dose-dependently and induced arrhythmic beats at the high concentration [19], therefore, we selected ouabain and digoxin to test our hypothesis.

As expected, both ouabain and digoxin dosedependently increased $\mathrm{Ca}^{2+}$-wave amplitude, reduced $\mathrm{Na}^{+}$-spike amplitude, and induced arrhythmia ("VT and VF-like" beats) in hiPSC-CMs (Fig. 1-3). The induction of altered $\mathrm{Na}^{+}$and $\mathrm{Ca}^{2+}$ cycling by both cardiac glycosides created a proarrhythmic state. The significant shortening of FPDcf by ouabain and digoxin was consistent with their effects to reduce QT interval in the clinic $[20,21]$ and in the isolated guinea pig hearts (Fig. 4).
To examine the functional significance of increased $\mathrm{Ca}^{2+}$-wave amplitude, ouabain was also tested in the guinea pig Langendorff isolated hearts, a well-established ex vivo safety pharmacology model with demonstrated predictive value to the clinical outcome $[16,22,23]$. Ouabain increased ventricular contractility dosedependently, and arrhythmia (VT and VF) developed at higher concentrations. The correlation between changes of $\mathrm{Ca}^{2+}$-wave amplitude in hiPSC-CMs and that of maximal $+\mathrm{dP} / \mathrm{dt}$ in isolated hearts (Table 2) strongly suggested that $\mathrm{Ca}^{2+}$-wave amplitude may serve as an in vitro surrogate of contractility modulation.

To further evaluate the translation of $\mathrm{Ca}^{2+}$-wave amplitude to myocardial contractility, nifedipine was then tested in both iPSC-CMs and isolated hearts. Nifedipine was found to reduce $\mathrm{Ca}^{2+}$-wave amplitude and shortened FPDcf dose-dependently (Fig. 5). This result confirmed the findings made by Halbach et al. (2003) that the slow wave of FPs after " $\mathrm{Na}^{+}$-spike" was mainly mediated by $\mathrm{Ca}^{2+}$ influx [17]. Nifedipine accelerated the spontaneous beat rate of hiPSC-CMs dose-dependently, which was consistent with previously reported results [24]. Importantly, nifedipine dose-dependently reduced in both contractility (LVDP and $+\mathrm{dP} / \mathrm{dt}$ ) and QTcf in the isolated hearts (Fig. 6). The positive correlation of nifedipineinduced changes in $\mathrm{Ca}^{2+}$-wave amplitude and FPDcf in hiPSC-CMs versus those of $+\mathrm{dP} / \mathrm{dt}$ and QTcf in isolated hearts (Table 3) demonstrates the utility of $\mathrm{Ca}^{2+}$-wave amplitude and FPD to accurately predict the modulation of ventricular contractility and QT interval in an intact heart.

The blockade of L-type $\mathrm{Ca}^{2+}$ channel with a compound like nifedipine can mitigate the cardiotoxicity induced by cardiac glycosides $[25,26]$. Thus, if the increased $\mathrm{Ca}^{2+}$-wave amplitude reflects the $\mathrm{Ca}^{2+}$-overload in hiPSC-CMs induced by ouabain and digoxin, nifedipine would be predicted to attenuate this effect. In this study, concurrent application or pretreatment of hiPSC-CMs with nifedipine at $30 \mathrm{nM}$ significantly antagonized the elevation of $\mathrm{Ca}^{2+}$-wave amplitude (Fig. 7) and prevented the induction of arrhythmic beats by both ouabain and digoxin.

Guinea pigs are frequently relied upon to investigate the pharmacology and safety pharmacology of drugs as a surrogate for human cardiovascular effects as cardiac parameters such as contractility, repolarization, and arrhythmic events are readily discerned [27]. In the presented study, Langendorff preparations of guinea pig hearts were used to validate the findings observed with MEA analysis in hiPSC-derived cardiomyocytes.

Guo/Qian/Abrams/Tang/Weiser/Sanders/Kolaja 
Our analysis confirmed that the FPD from an MEA recording in cardiomyocytes is reminiscent of action potential duration (APD) seen in single cardiomyocyte preparations [17]. While pacing was not conducted, FPD is anticipated to behave in a manner analogous to APD and QT interval. Thus, FPDs were corrected for the alteration in the observed spontaneously beating rate. Although Bazett's correction formula is widely used clinically and has been previously applied in stem cellderived cardiomyocytes [8], Fridericia's formula was selected in this study to correct for the widely-ranging change of the beat rate caused by ouabain, digoxin and nifedipine, to avoid correction errors seen with Bazett's formula due to large increases in both high and low baseline heart rates [28].

The finding that nifedipine increased the beat rate in monolayers of human embryonic stem cell-derived cardiomyocytes was also observed by Satin et al. [24]. Unlike an intact heart where the beating rhythm is tightly controlled by the specialized pacemaker cells which rely on L-type $\mathrm{Ca}^{2+}$ channels to contribute to the spontaneous depolarization in initiating the cardiac action potential, spontaneously beating hiPSC-CMs could rely on $\mathrm{Na}^{+}$ channels to initiate the action potential with $\mathrm{L}$-type $\mathrm{Ca}^{2+}$ channels contributing to the plateau phase [24]. In this preparation, treatment with nifedipine leads to an increased beat rate by shortening of the duration of the action potential.

\section{Conclusion}

Our data supports the use of hiPSC-CMs with MEAs to study in vitro electrophysiological effects in a model suitable for early cardiac drug safety evaluation. Our study expands the use of this model to identify proarrhythmic risks not associated with $\mathrm{hERG}$ inhibition and/or QT liability, but stemming from dysregulated $\mathrm{Na}^{+}$and $\mathrm{Ca}^{2+}$-cycling such as that induced by cardiac glycosides. Proof-of-concept evidence is presented demonstrating the capability of this model to detect cardiac liabilities of drugs on both ion channels and ion transporters.

\section{Acknowledgements}

The authors would like to acknowledge Thomas Singer for his managerial support of this project. In addition, the authors would like to thank Laura Suter-Dick and Blake Anson for review of the manuscript, and Jalpa Modi and Cheryl Heidelberger for technical assistance.

\section{References}

1 Kola I: The state of innovation in drug development. Clin Pharmacol Ther 2008:83:227-30.

2 ICH guidance S7A and S7B: http:// private.ich.org/cache/compo/502-2721.html.

-3 Krewski D, Acosta D Jr, Andersen M, Anderson H, Bailar JC 3rd, Boekelheide K, Brent R, Charnley G, Cheung VG, Green S Jr, Kelsey KT, Kerkvliet NI, Li AA, McCray L, Meyer O, Patterson RD, Pennie W, Scala RA, Solomon GM, Stephens M, Yager J, Zeise L: Toxicity testing in the 21 st century: a vision and a strategy. J Toxicol Environ Health B Crit Rev 2010:13:51-138.

-4 MacDonald JS, Robertson RT: Toxicity testing in the 21 st century: a view from the pharmaceutical industry. Toxicol Sci 2009; 110:40-46.
Kattman SJ, Koonce CH, Swanson BJ, Anson BD: Stem cells and their derivatives: a renaissance in cardiovascular translational research. J Cardiovasc Transl Res 2011;4:66-72.

Phillips BW, Crook JM: Pluripotent human stem cells: A novel tool in drug discovery. BioDrugs 2010;24:99-108.

Dick E, Rajamohan D, Ronksley J, Denning C: Evaluating the utility of cardiomyocytes from human pluripotent stem cells for drug screening. Biochem Soc Trans 2010;38:1037-1045.

Caspi O, Itzhaki I, Kehat I, Gepstein A, Arbel G, Huber I, Satin J, Gepstein L: In vitro electrophysiological drug testing using human embryonic stem cell derived cardiomyocytes. Stem Cells Dev 2009; 18:161-72.
Yokoo N, Baba S, Kaichi S, Niwa A, Mima T, Doi H, Yamanaka S, Nakahata T, Heike T: The effects of cardioactive drugs on cardiomyocytes derived from human induced pluripotent stem cells. Biochem Biophys Res Commun 2009;387:482-488.

Zwi L, Caspi O, Arbel G, Huber I, Gepstein A, Park IH, Gepstein L: Cardiomyocyte differentiation of human induced pluripotent stem cells. Circulation 2009;120:1513-1523.

11 Meyer T, Boven KH, Günther E, Fejtl $\mathrm{M}$ : Micro-electrode arrays in cardiac safety pharmacology: a novel tool to study QT interval prolongation. Drug Saf 2004;27:763-772. 
12 Liang H, Matzkies M, Schunkert H, Tang M, Bonnemeier H, Hescheler J, Reppel $\mathrm{M}$ : Human and murine embryonic stem cell-derived cardiomyocytes serve together as a valuable model for drug safety screening. Cell Physiol Biochem 2010;25:459-66.

$>13$ Asai Y, Tada M: Combination of functional cardiomyocytes derived from human stem cells and a highly-efficient microelectrode array system: an ideal hybrid model assay for drug development. Curr Stem Cell Res Ther 2010;5:22732.

14 Demiryürek AT, Demiryürek S: Cardiotoxicity of digitalis glycosides: roles of autonomic pathways, autacoids and ion channels. Auton Autacoid Pharmacol 2005;25:35-52.

$>15$ Farkas AS, Nattel S: Minimizing repolarization-related proarrhythmic risk in drug development and clinical practice. Drugs 2010;70:573-603.

$>16$ Guo L, Dong Z, Guthrie H: Validation of a guinea pig Langendorff heart model for assessing potential cardiovascular liability of drug candidates. J Pharmacol Toxicol Methods 2009;60:130-51.

17 Halbach M, Egert U, Hescheler J, Banach $\mathrm{K}$ : Estimation of action potential changes from field potential recordings in multicellular mouse cardiac myocyte cultures. Cell Physiol Biochem 2003;13:271-84.
18 Pasquié JL, Richard S: Prolongation in QT interval is not predictive of $\mathrm{Ca}^{2+}$ dependent arrhythmias: implications for drug safety. Expert Opin Drug Saf 2009;8:57-72.

19 Qian JY, Guo L: Altered cytosolic $\mathrm{Ca}^{2+}$ dynamics in cultured Guinea pig cardiomyocytes as an in vitro model to identify potential cardiotoxicants. Toxicol In Vitro 2010;24:960-72.

20 Lauten A, Abmann I, Knappe J: Acute electrophysiological effects of cardiac glycosides on sinus node function and ventricular repolarization under clinical conditions Cor Vasa 1987;29:436-43.

21 Saner HE, Lange HW, Pierach CA, Aeppli DM: Relation between serum digoxin concentration and the electrocardiogram. Clin Cardiol 1988;11:752-756.

-2 2 Wu L, Shryock JC, Song Y, Li Y, Antzelevitch C, Belardinelli L: Antiarrhythmic effects of ranolazine in a guinea pig in vitro model of long-QT syndrome. J Pharmacol Exp Ther 2004;310:599-605.

23 Foster NJ, Haines DE: The antiarrhythmic effects of ranolazine. Rev Cardiovasc Med 2009;10:S38-45.
Satin J, Kehat I, Caspi O, Huber I, Arbel G, Itzhaki I, Magyar J, Schroder EA, Perlman I, and Gepstein L: Mechanism of spontaneous excitability in human embryonic stem cell derived cardiomyocytes. J Physiol 2004;559:479-496.

25 Bush LR, Evans RM, Gaul SL, D’Alonzo AJ: Comparative effects of verapamil, diltiazem and felodipine during experimental digitalis-induced arrhythmias. Pharmacology 1987;34:111-20.

-26 Khatter JC, Agbanyo M, Hoeschen RJ, Navaratnam S, Bains R: Digitalis-induced mechanical toxicity: protection by slow $\mathrm{Ca}++$ channel blockers. J Pharmacol Exp Ther 1986;239:206-10.

27 Hauser DS, Stade M, Schmidt A, Hanauer $\mathrm{G}$ : Cardiovascular parameters in anaesthetized guinea pigs: a safety pharmacology screening model. J Pharmacol Toxicol Methods 2005:52:106-14.

28 Guidance for Industry: E-14 "Clinical evaluation of QT/QTc interval prolongation and proarrhythmic potential for non-antiarrhythmic drugs". http:// w w w. fda.gov/dow n load s/ RegulatoryInformation/Guidances/ ucm 129357.pdf 